\title{
A Microstructural Map of Crystallized NiTi Thin Film Derived from In Situ TEM Methods
}

\author{
Hoo-Jeong Lee ${ }^{1}$, Hai $\mathrm{Ni}^{2}$, David T. $\mathrm{Wu}^{2}$ and Ainissa G. Ramirez ${ }^{2, *}$ \\ ${ }^{1}$ School of Advanced Materials Science and Engineering, Sungkyunkwan University, Kyongki-Do, Korea \\ ${ }^{2}$ Department of Mechanical Engineering, Yale University, New Haven, Connecticut, 06520, USA
}

Sputtered-deposited nickel titanium thin films are commonly amorphous when synthesized and require annealing to crystallize them. The resulting microstructures, which are governed by nucleation and growth kinetics, dictate the actuation properties. The evolution of these microstructures was studied using in situ transmission electron microscopy (TEM) heating methods. The experimentally-determined kinetic values of nucleation and growth were inserted into a mathematical expression derived from the Johnson-Mehl-Avrami-Kolmogorov (JMAK) theory, which predicts the average grain size over a broad range of temperatures.

(Received September 26, 2005; Accepted February 7, 2006; Published March 15, 2006)

Keywords: thin films, shape memory alloys, NiTi, in situ transmission electron microsopy (TEM), martensitic transformation, crystallization, kinetics, Avrami model

\section{Introduction}

Nickel titanium has been of technological interest for more than several decades because it is an excellent candidate for actuation resulting from its shape memory properties. Fabricating it in the thin film form has recently become of interest, particularly in the development of MEMS devices because these films provide the highest actuation forces compared to other actuation modes (i.e. electrostatic, thermal, and piezoelectric). ${ }^{1)}$ These films will benefit applications, such as microvalves, micropumps, and micromanipulators, where large displacement forces are required..$^{2,3)}$ The actuation properties of these materials are based on a martensitic phase transformation, or a displacive shift in atomic crystalline structure. ${ }^{4,5)}$ Consequently, the control of this transformation and its associated mechanical properties are important issues in order to create reliable and controllable devices.

It has been suggested that there is a link between the microstructural grain size and the martensitic transformation of shape memory alloys. ${ }^{6)}$ Possible strain constraints imposed by grain boundaries have been thought to play an important role during twinning in these martensitic transformations. ${ }^{6,7)}$ There has been earlier experimental work in bulk materials to reveal such a connection. ${ }^{7-9)}$ However, the controllability of the grain size has been limited in the bulk because a multistep process is needed to achieve various grain sizes, including cold rolling and subsequent recrystallization annealing steps. ${ }^{7,9)}$ These processing methods depend on heavy mechanical deformation and grain growth at high temperatures, which can introduce other adverse effects. Although these earlier studies hint at a connection between the grain size and the transformation behavior and a connection between the grain size and twinning mode, the direct link between the grain size and martensitic transformation largely remains unproven.

Thin films offer a much better opportunity than bulk to explore this connection. Unlike their bulk counterparts,

*Corresponding author, E-mail address: ramirez@stanfordalumni.org nickel titanium thin films are commonly sputtered-deposited in an amorphous form and require a high-temperature annealing step to create their crystalline (actuating) form. ${ }^{10,11)}$ Since the initial microstructure is a featureless amorphous structure, the resulting microstructure is solely dependant on the conditions of annealing. This unique condition places us a much better position to control the grain size. In this case, the microstructure control hinges solely on understanding the crystallization process, which is a wellmodeled process of materials transformations. Provided that the details of the kinetic events that occur during crystallization are known, we should be able to control the microstructure simply by altering annealing parameters such as time and temperature. This unique position motivated us to embark on the study to characterize the crystallization process of amorphous NiTi thin films and discover processing methods to control the grain size.

The goal of this paper is to systematically study the crystallization of nickel titanium thin films and determine the rates of nucleation and growth as a function of temperature. With this information, we acquire a full description of crystallization. In addition, knowing these rates allows us to use a crystallization theory in a new and effective way, that is, to predict the resulting microstructures for a given processing conditions. NiTi shows that its crystallization behavior is uncomplicated and straightforward to model because it crystallizes polymorphically (that is, a phase transformation where only the structure changes and its composition remains the same). Leveraging this ideal crystallization behavior with a method to fully describe the nucleation and growth rates, we are able to glean new information about the development of its microstructure. Such information will provide the groundwork for controlling phase transformations in thin film NiTi and their associated actuation properties prescribed by microstructures.

\section{Experimental Methods}

We synthesized amorphous NiTi films, $0.2 \mu \mathrm{m}$ thick, in a $2.4 \mathrm{~Pa}$ argon-filled dc magnetron sputtering chamber (with a 
base pressure of $2.0 \times 10^{-7} \mathrm{~Pa}$ ). Two 75-mm targets of NiTi and $\mathrm{Ti}$ (at powers of 300 and $89 \mathrm{~W}$ ) generated a deposition rate of $0.76 \mathrm{~nm} / \mathrm{s}$. These films were deposited onto novel micromachined silicon-nitride TEM membrane samples, ${ }^{13,14)}$ which were pivotal to this study because they possess several advantages over traditional wedge-style ionmilled samples: They provide a rigid support, a large view area (of $1 \mathrm{~mm}^{2}$ ), and a constant thickness, which ensures a uniform temperature profile. ${ }^{13)}$ The chemical composition of 50.4 at $\% \mathrm{Ti}$ and $49.6 \mathrm{at} \% \mathrm{Ni}$ was determined by electron microprobe, which was calibrated with standards of known compositions.

Real-time in situ heating studies were performed in a bright-field mode on a transmission electron microscope equipped with a field-emission gun operating at $200 \mathrm{kV}$. Samples were heated within the microscope on a single-tilt heating holder with heating capabilities of up to $1300^{\circ} \mathrm{C}$. We controlled the temperature manually by adjusting the voltage at a constant current, while measuring the temperature with a thermocouple. To insure thermal uniformity across the TEM membrane, samples were heated initially at a rate of $30^{\circ} \mathrm{C} /$ min until they reached $445^{\circ} \mathrm{C}$. At that point, the temperature was quickly raised to target temperatures (of 465, 475, 495, 505 , and $515^{\circ} \mathrm{C}$ ) and held constant until the crystallization process was complete. In order to examine a wide field of view, a low magnification was used, and the smallest observable feature (or spatial resolution) was approximately $50 \mathrm{~nm}$. Digital images of the samples were recorded on videotapes at intervals of two seconds through a CCD camera. Each image was time-stamped, which allowed us to know precisely when events occurred. We estimated the nucleation rate and the growth rate by analyzing scores of crystallites in dozens of images. The growth rate was calculated by measuring the change in crystal size. We measured the nucleation rate by counting the number of nucleation sites as a function of time. ${ }^{14}$

\section{Results and Discussions}

Crystallization can be thought of as a combination of steps: nucleation of crystals from amorphous state and the growth of these crystals. There is also an interaction of these crystals to consider (i.e. impingement). This overall kinetics of crystallization has been modeled by the Johnson-MehlAvrami-Kolmogorov (JMAK) theory, which is a widelyaccepted for phase transformation of materials. ${ }^{15)}$ It describes the process by using the crystallized area fraction $\Phi$ as a function of time, and is expressed as:

$$
\Phi=1-\exp \left(-k t^{n}\right)
$$

where $k$ is a rate constant, which is dependent on temperature, and $n$ is the Avrami exponent, which is dependent on the dimensionality of growth and the time-dependence of both nucleation and growth.

For a thin film undergoing nucleation and growth, the JMAK theory predicts that the number of grains per unit area at the end of the transformation $(\mathrm{N})$ is

$$
N=\int_{0}^{\infty} J(t)(1-\Phi(t)) d t
$$

where $J$ is the nucleation rate $\left(\# / \mathrm{m}^{2} \mathrm{~s}\right)$ and $\Phi$ is the area fraction transformed. If we assume that $J$ and $V$, the growth rate, are constant with respective to time, $J_{0}$ and $V_{0}$, then the number of grains can be simplified to:

$$
N=J_{0} \int_{0}^{\infty} \exp \left\{-\pi J_{0} V_{0}^{2} t^{3} / 3\right\} d t
$$

By substituting a new integration variable, $x=\pi J_{0} V_{o}^{2} t^{3} / 3$, then Eq. (3) becomes

$$
N=J_{0} \int_{0}^{\infty} e^{-x} \frac{d t}{d x} d x=\frac{J_{0}}{3}\left(\frac{3}{\pi J_{0} V_{0}^{2}}\right)^{\frac{1}{3}} \int_{0}^{\infty} x^{\frac{1}{3}-1} e^{-x} d x
$$

If we note that the gamma function is defined as $\Gamma(u)=$ $\int_{0}^{\infty} x^{u-1} e^{-x} d x$, and note that the inverse of this expression is the mean area per grain, we get the expression,

$$
\langle A\rangle=\frac{1}{N}=\frac{(9 \pi)^{1 / 3}}{\Gamma(1 / 3)}\left(\frac{V_{0}}{J_{0}}\right)^{2 / 3} \approx 1.137\left(\frac{V_{0}}{J_{0}}\right)^{2 / 3}
$$

Assuming disc-shaped grains, the average diameter of grains can be approximated as

$$
\langle d\rangle \approx 2\left(\frac{\langle A\rangle}{\pi}\right)^{1 / 2} \approx 1.2\left(\frac{V_{0}}{J_{0}}\right)^{1 / 3}
$$

From this simple expression, we confirm that a good estimation of the grain size is possible if the steady-state nucleation and growth rates are known. The values of the nucleation and growth rates and their dependence on temperature and time can be most effectively obtained from real-time observations of the crystallization process. In this paper, we used in situ transmission electron microscopy heating methods to observe this process and to attain kinetic parameters. Figure 1 shows electron micrographs taken during such an in situ observation. Before heating, all samples exhibited a gray color and were absent of features, which indicate an amorphous microstructure. We heated a sample to $495^{\circ} \mathrm{C}$. Once the target temperature was reached, samples were held for an incubation time, after which crystals began appearing in the gray matrix as small spots with bright or dark contrast [Fig. 1(a)]. The difference in
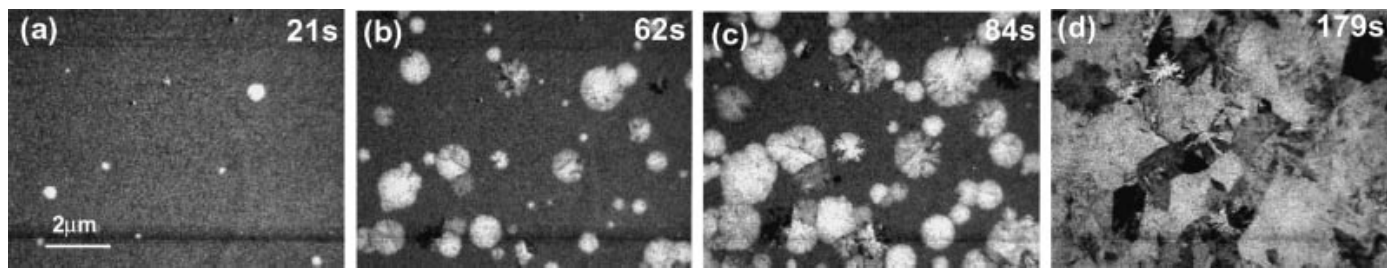

Fig. 1 Video-captured TEM bright-field images taken during in situ heating that show the progression of crystallization in amorphous nickel-titanium films at $490^{\circ} \mathrm{C}$. Each image was taken after the onset of nucleation at times of: (a) $21 \mathrm{~s}$; (b) $62 \mathrm{~s}$; (c) $84 \mathrm{~s}$; and (d) $179 \mathrm{~s}$. 
(a)

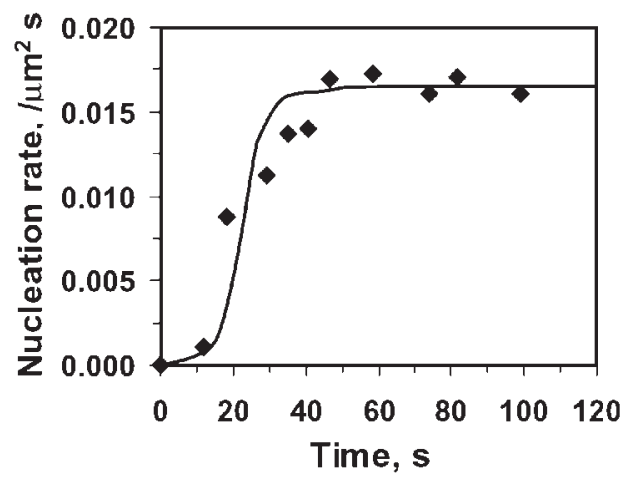

(b)

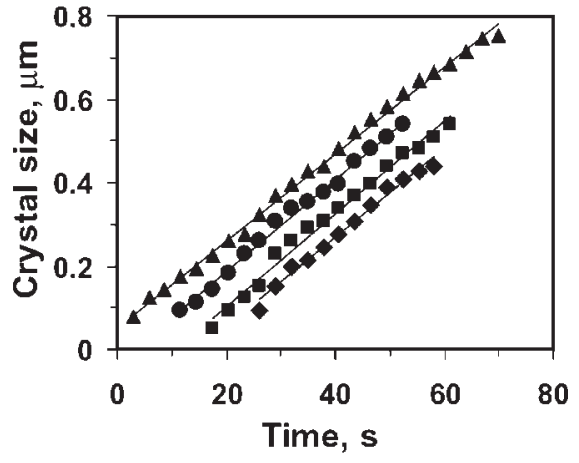

Fig. 2 (a) The nucleation rate, $J$, as a function of time, exhibits a sigmoidal (or S-shaped) behavior and reaches its steady-state within $25 \mathrm{~s}$ (less than $15 \%$ of the of the total crystallization time of $180 \mathrm{~s}$ ). (b) A plot of crystal size (which is proportional to the growth rate) taken at different times. It exhibits a linear behavior indicating that the growth rate is constant over time.

contrast in these spots is due to the interaction of the plane orientation and the direction of the electron beams. With continuous heating at these target temperatures, these initial spots (or grains) grew quickly into large circular crystals, while new spots steadily populated the remaining untransformed area during the entire crystallization process, as depicted in Fig. 1(b). These crystals grew isotropically until impingement with other crystals until the crystallization is complete [Figs. 1(c) and (d)].

We analyzed dozens images from video recordings of such in situ heating experiments and were able to obtain a quantitative understanding of the temporal behavior of the nucleation and growth rates. We estimated the nucleation and growth rate by tallying the number of grains and their size in dozens of micrographs. The number of images used for each temperature ranged from 15 to 30 , except for the high temperature sample, which crystallized rapidly and could only be captured in 5 frames. To determine the nucleation rate, $J$, we determined at the time event of $t_{i}$, the number of crystals in each micrograph $N\left(t_{i}\right)$. Using the transformed area fraction, $\Phi\left(t_{i}\right)$, we can express the nucleation rate as:

$$
J\left(t_{i}\right)=\frac{1}{1-\Phi\left(t_{i}\right)} \frac{d N\left(t_{i}\right)}{d t} \approx \frac{1}{1-\Phi\left(t_{i}\right)} \frac{N\left(t_{i}\right)-N\left(t_{i-1}\right)}{t_{i}-t_{i-1}}
$$

We plotted the nucleation rate, $J$, as a function of time and found that it had a typical sigmoidal or S-shape curve, as shown in Fig. 2(a). This curve shows a low rate of increase that quickly ramps upward, and then finally plateaus to a steady-sate. Steady state was mostly reached in a relatively short time, typically within $15 \%$. We estimated the steadystate value of the nucleation rate, $J_{0}$, by averaging the values of $J$ at the plateau.

We also determined the growth rate of the crystals, $V$, by measuring the size of each crystal in each micrograph and tracking the change as a function of time. A contour was drawn around each crystal and the area was computed. From the area of the crystal, a nominal diameter was determined using the ASTM method $(d=\sqrt{4 \pi A}) .{ }^{7}$,14) As a function of time, we observed a constant growth rate throughout the transformation, regardless of the crystal size. This seems to suggest that the transformation is non-diffusional and that coarsening (or Ostwald ripening) is not active.

To investigate the effects of temperature on the crystal-



Fig. 3 JMAK plots for the crystallization of amorphous NiTi thin films annealed at a range of different temperatures (from 738 to $788 \mathrm{~K}$ ).

lization process, we examined the microstructure development and the time of crystallization for samples heated to $465,475,495,505$, and $515^{\circ} \mathrm{C}$. The total crystallization time decreased drastically from $4700 \mathrm{~s}$ for the lowest temperature $\left(465^{\circ} \mathrm{C}\right)$ to $11 \mathrm{~s}$ for the highest temperature. Figure 3 shows the dependence of crystallized area fractions with respect to time taken from in situ observations for those samples. The analysis of in situ observations also reveals that the nucleation and growth rates varied drastically with temperature: there were many more nuclei formed at higher temperatures, and their growth was much more rapid at high temperatures. We also found that both the nucleation and growth rates were higher at higher temperatures. These overall trends governed the final microstructure and gives rise to significantly different microstructures even over a small temperature range.

Figure 4 shows the temperature dependence of the nucleation and growth rates for our samples. It can be thought, within a limited temperature range, that the nucleation rate and growth rate have Arrhenius behaviors, where $J=J^{*} \exp (-H / k T)$ and $V=V^{*} \exp (-E / k T) .{ }^{16)}$ The terms $H$ and $E$ are the effective activation energies for nucleation and growth. As Fig. 4 shows, our steady-state nucleation rate and growth rate also exhibit an Arrhenius temperature dependence, which agrees with other studies in 

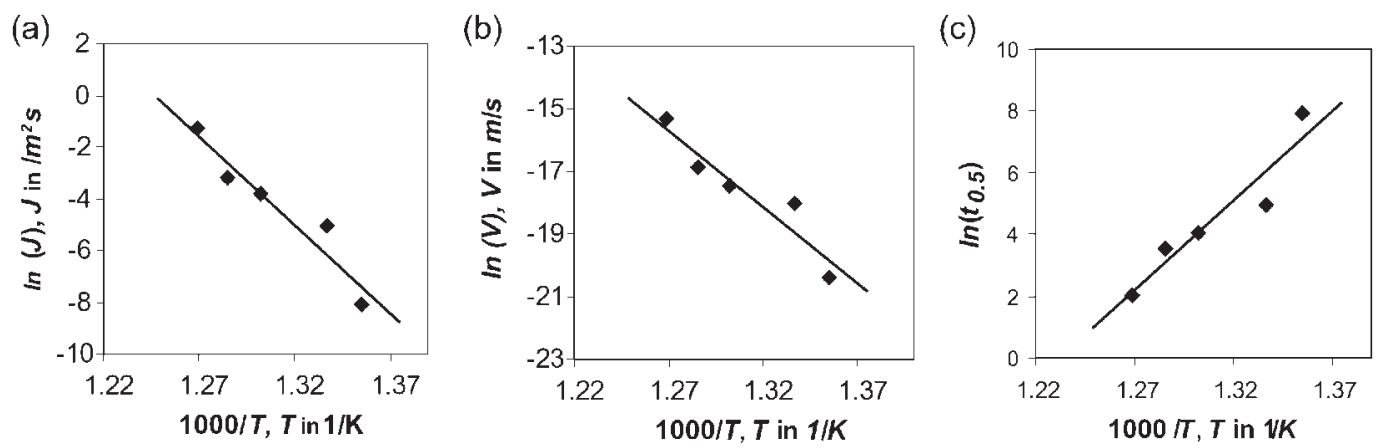

Fig. 4 Arrhenius plots of: (a) the nucleation and (b) growth rates, and (c) the overall crystallization.

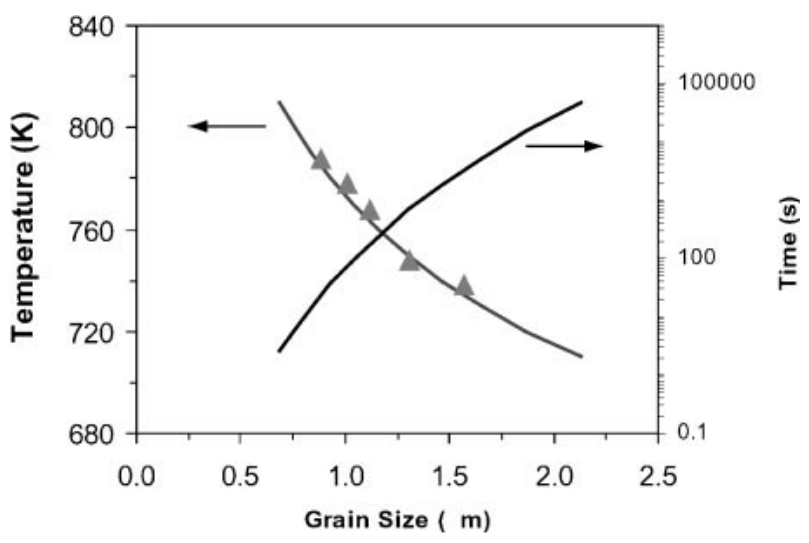

Fig. 5 A microstructure map of crystallized NiTi films. This graph shows a relationship between a grain diameter and annealing conditions (of temperature and time).

the literature. ${ }^{16)}$ The activation energies for nucleation and growth are the slopes, which are 567.5 and $404.8 \mathrm{~kJ} / \mathrm{mol}$ respectively. In addition to this, Fig. 4(c) displays the temperature dependence of the overall crystallization. By plotting $\ln \left(t_{0.5}\right)$ vs. $1 / T$, where $t_{0.5}$ is the time at $50 \%$ transformation, we get the temperature dependence of the overall crystallization process. It, too, follows an Arrhenius behavior and can be curve-fitted and the slope is $\mathrm{W}$, the overall crystallization activation energy, which was found
$475.6 \mathrm{~kJ} / \mathrm{mol}$. From this, we can determine total time needed for the completion of the crystallization process $\left(2 t_{0.5}=t_{f}\right)$.

With the full description of nucleation and growth with respect to temperature and time, we can solve eq. (5) to obtain an expression for grain size as a function of temperature and time. Plugging the linear relations estimated from Fig. 5 into eq. (5) produces a relationship for grain size at various annealing temperatures. From $t_{f}=2 t_{0.5}$, we can also estimate total time needed for the crystallization for each temperature. These combined values provide us with a microstructure map for crystallization processes, as seen in Fig. 5. From this graph, optimum processing conditions of annealing temperature and time for grains of a desired size can be effectively chosen a priori. The triangles in the graph correspond to experimentally measured grain sizes for samples annealed at a few selected temperatures. Their closeness to the predicted line demonstrates the robustness of our approach in generating a description of individual kinetic steps, which, in turn, rendered us able to predict the resulting grain size at varying annealing temperatures. ${ }^{17)}$

Our preliminary work indicates that the grain size is effectively controlled by following the microstructure map and that the strength of the transformation is indeed strongly dependant on the grain size. Shown in Fig. 6 are orientation contrast electron micrograph taken in a scanning electron microscope (SEM) from this work. In the micrographs, surface marks produced by martensitic transformation were
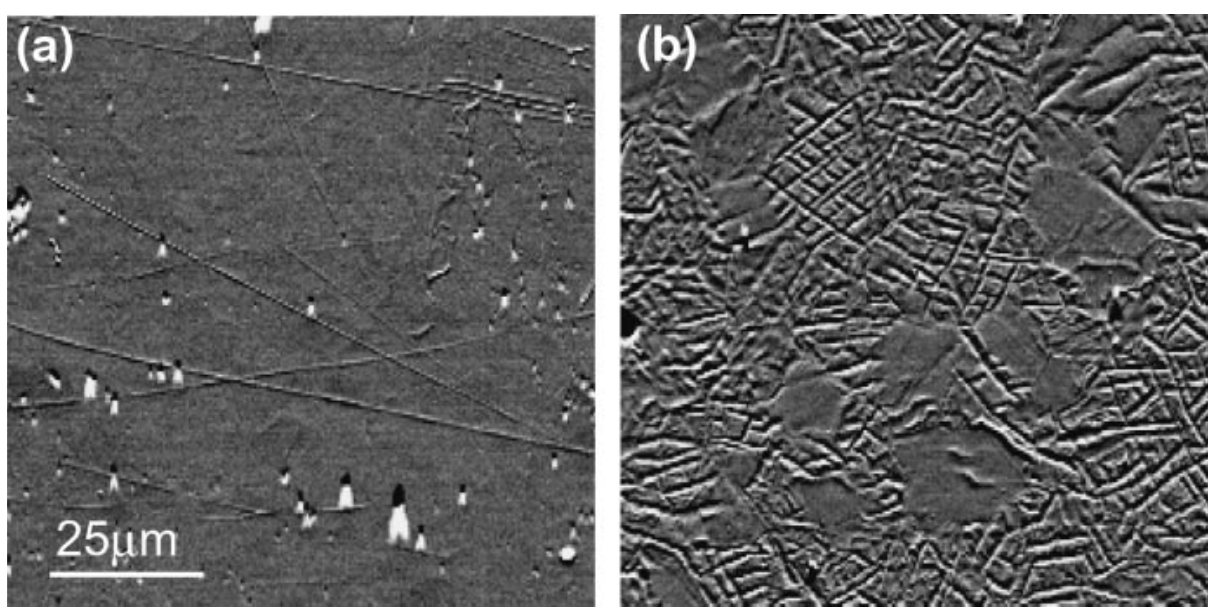

Fig. 6 Orientation contrast electron micrographs taken from SEM for samples which were crystallized using the conditions of the microstructure map shown in Fig. 5. The samples shown were annealed (a) at $788 \mathrm{~K}$ for $1 \mathrm{~min}$ and (b) at $738 \mathrm{~K}$ and $2 \mathrm{~h}$. Lines with a dark contrast indicate surface marks caused by martensitic transformation. 
heightened in contrast due to the effects of backscattered electrons channeling in and out through lattice planes. These micrographs clearly show that the density of surface marks are drastically different for two samples annealed at different conditions, one at $788 \mathrm{~K}$ for $1 \mathrm{~min}$ and the other at $738 \mathrm{~K}$ for $2 \mathrm{~h}$. According to the microstructure map, the latter should have a much larger grain size. The density of the marks appears much higher in the sample with a larger grain size than in a small grain sample, which indicates that the martensitic transformation has occurred much stronger in the larger grain sample. This evidence underscores the utility in controlling the grain size and in predicting the actuation behavior of materials, which are connected to the microstructure. As such, this ability to predict grain size has practical applications in the design of thin film for applications, such as MEMS.

\section{Summary}

In this study, we explored an experimental method to control the grain size during the crystallization of NiTi amorphous thin films. A simple mathematical treatment of the Johnson-Mehl-Avrami-Kolmogorov (JMAK) theory generated a simple expression for the grain size, which depends only on temperature and time of crystallization. Real-time in situ TEM methods were adopted to effectively capture such information. By capturing the values of nucleation and growth at different temperatures, we are able to solve the equation derived from the JMAK theory. This generated a powerful microstructure map, which can provide annealing conditions (time and temperature) for a desired grain size. Our preliminary data also confirms that the samples produced with different grain size follow the microstructure map and show significantly different martensitic transformation behavior.

\section{Acknowledgements}

This work was supported by US NSF grant No. 0347095.

\section{REFERENCES}

1) P. Krulevitch, H. Bornhauser, T. Hochuli et al.: Sens. Actuators, A 2123 (1990) 247-252.

2) A. Ishida and V. Marynov: MRS Bulletin (2002) 111.

3) W. L. Bernard, H. Kahn, A. H. Heuer et al.: Journal of Microelectromechanical Systems 7 (1998) 245.

4) K. Bhattacharya, S. Conti, G. Zanzotto et al.: Nature 428 (6978) (2004) 55.

5) C. M. Wayman and T. W. Duerig: Engineering Aspects of Shape Memory Alloys, ed. by T. W. Duerig, K. N. Melton, D. Stockel et al. (Butterworth-Heinemann, New York, 1990).

6) K. Bhattacharya, Microstructure of Martensite (Oxford University Press, New York, 2003).

7) M. Nishida, I Itai, K. Kitamura et al.: J. Phys. IV 5 (C8) (1995) 635.

8) F. J. Gil, J. M. Manero and J. A. Planell: J. Mater. Sci. 30 (1995) 2526.

9) Y. Motohashi, K. Ohsawa, T. Hoshiya et al.: J. Jpn. Inst. Metals 55 (1991) 132.

10) J. J. Kim, P. Moine and D. A. Stevenson: Scr. Metall. 20 (1986) 243.

11) J. D. Busch, A. D. Johnson, C. H. Lee et al.: J. Appl. Phys. 68 (1990) 6224.

12) Hai Ni, Hoo-Jeong Lee, and Ainissa G. Ramirez: Sens. Actuators, A 119 (2005) 553.

13) H.-J. Lee and A. G. Ramirez: Appl. Phys. Lett. 85 (2004) 1146.

14) ASTM Designation E112.

15) J. W. Christian: The Theory of Transformation in Metals and Alloys, Second ed. (Pergamon, New York, 1975).

16) M. G. Scott: Amorphous Metallic Alloys, ed. by F. E. Luborsky (Butterworths, London, 1983).

17) H.-J. Lee, H. Ni, D. T. Wu et al.: Appl. Phys. Lett. 87 (2005) 124102. 